\title{
Variación estacional de la diversidad de coleópteros epigeos en la Laguna Carén (Santiago, Chile)
}

\author{
Seasonal variation of the epigeal coleopteran diversity in the Laguna Carén (Santiago, Chile)
}

\author{
ALEXANDRA ALVARADO-ORELLANA ${ }^{1}$, AMANDA HUERTA-FUENTES $^{2}$, ÁLVARO PALMA-MUÑOZ ${ }^{3}$ \\ y SEBASTIÁN RODRÍGUEZ-TOBAR ${ }^{4}$
}

\begin{abstract}
Resumen: Se evaluó la variación estacional de la diversidad de coleópteros epigeos y de parámetros físico-químicos asociados a la calidad del agua en la Laguna Carén (Santiago, Chile). Se midieron en primavera y verano diversos parámetros físico-químicos del agua de la laguna en cuatro estaciones de muestreo aleatorias, representativas de las coberturas vegetales. Los coleópteros epigeos de la ribera se recolectaron mediante trampas de intercepción $(n=40)$, instaladas al azar. Se estimó la abundancia y riqueza de los coleópteros por estación de muestreo y temporada. Además, se separaron en gremios tróficos: fitófagos, depredadores y saprófagos. Las diferencias de la abundancia y riqueza se evaluaron con pruebas no paramétrica de Kruskal-Wallis, seguidas por pruebas de Dunn (por estaciones de muestreo) y de Mann-Whitney (por temporada). El mismo procedimiento estadístico se aplicó a los gremios tróficos. El ensamble de especies de coleópteros epigeos estuvo representado por 19 especies (14 de ellas nativas) provenientes de ocho familias, constituyéndose en el primer registro de insectos en esta laguna. La riqueza de los coleópteros epigeos fue significativamente superior en primavera, en tanto que la abundancia lo fue en verano, coincidiendo con las estaciones de muestreo con cobertura vegetal más alta. La disponibilidad de alimento y refugio para los coleópteros epigeos estaría influenciada principalmente por factores antrópicos, que conducen a un mayor grado de fragmentación y alteración del hábitat. La información generada podrá servir como base para futuras investigaciones sobre bioindicadores en humedales vulnerables de la región mediterránea.
\end{abstract}

Palabras clave: Abundancia, riqueza, depredadores, fitófagos, saprófagos.

\begin{abstract}
The seasonal variation of epigeal coleopteran diversity and physical-chemical parameters associated to water quality in the Laguna Carén (Santiago, Chile) was evaluated. Different physical-chemical parameters of lagoon water were measured in spring and summer at four random sampling stations, representative of the vegetable coverages. The epigeal coleopterans of the shore were collected by means of interception traps $(n=40)$, installed at random. It was estimated the abundance and richness of the coleopterans by sampling station and season. In addition, they were separated into trophic guilds: phytophagous, predatory and saprophagous. The differences in abundance and richness were evaluated with nonparametric Kruskal-Wallis tests, followed by Dunn (sampling station) and MannWhitney (season) tests. The same statistical procedure was applied to trophic guilds. The ensemble of species of epigeal coleopteran was represented by 19 species (14 natives) from eight families, constitutes the first record of insects in the lagoon. The richness of epigeal coleopteran was significantly higher in spring; while abundance was in summer, concurring with the sampling stations with higher coverages. The availability of food and shelter for epigeal beetles would be influenced mainly by anthropic factors, which lead to a greater degree of fragmentation and alteration of the habitat. The information generated can serve as a basis for future research on bioindicators in vulnerable wetlands in the Mediterranean region.
\end{abstract}

Key words: Abundance, richness, predators, phytophagous, saprophagous.

\section{Introducción}

Los recursos hídricos están expuestos a fenómenos naturales $\mathrm{y}$ actividades humanas que influyen en la disponibilidad de uso como en su calidad. La evaluación de la calidad del agua constituye un tema de gran relevancia, lo que ha llevado a la búsqueda de indicadores e índices que permitan una interpretación confiable de su estado real (The World Water Development Report, WWDR 2015).

En este sentido, en la valoración de la calidad del agua se han utilizado históricamente parámetros físico-químicos, que entregan sólo características inmediatas sobre el ecosistema (Figueroa et al. 2016). Los bioindicadores son especies o grupos taxonómicos capaces de reflejar el estado de con- servación, endemismo, diversidad, y grado de perturbación en los ecosistemas. Las especies indicadoras son aquellos organismos o sus restos que ayudan a interpretar cualquier fenómeno, acontecimiento pasado o actual relacionado con el estudio del medio. Ofrecen ventajas dado que se encuentran en casi la totalidad de los sistemas ecológicos, informan de la situación en el tiempo y favorecen estudios comparativos. Éstos deben ser en general, especies abundantes, sensibles, fáciles y rápidas de identificar, con poca movilidad y estudiadas, tanto en su ecología como en su ciclo. Los macroinvertebrados más utilizados pertenecen a la clase Insecta (Tenjo y Cárdenas 2015). Los insectos son los organismos más diversos y dominantes en los ecosistemas terrestres, ocupan una amplia variedad de hábitats, tienen ciclos biológicos cortos

${ }^{1}$ Ing. Forestal, Departamento de Silvicultura y Conservación de la Naturaleza, Facultad de Ciencias Forestales y Conservación de la Naturaleza, Universidad de Chile. Casilla 9206, Santiago, Chile, alexandra.alvarado@ug.uchile.cl. ${ }^{2}$ Dra. Ing. Forestal, Departamento de Silvicultura y Conservación de la Naturaleza, Facultad de Ciencias Forestales y Conservación de la Naturaleza, Universidad de Chile. Casilla 9206, Santiago, Chile, ahuerta@uchile.cl. ${ }^{3}$ Ing. en Recursos Naturales, Laboratorio de Criminalística Central, Policía de Investigaciones de Chile (PDI). Santiago, Chile, appalma.m@gmail.com. ${ }^{4}$ Biólogo, Instituto de Biología, Pontificia Universidad Católica de Valparaíso. Valparaíso, Chile, s.rodriguez.tobar@gmail.com. Autor para correspondencia. Amanda HuertaFuentes. Dra. Ing. Forestal, Departamento de Silvicultura y Conservación de la Naturaleza, Facultad de Ciencias Forestales y Conservación de la Naturaleza, Universidad de Chile. Casilla 9206, Santiago, Chile, ahuerta@uchile.cl. 
asociados a microhábitats y una elevada riqueza específica. Los insectos representan más del $85 \%$ de las especies vivientes y el $75 \%$ de los artrópodos conocidos, por lo cual constituyen el grupo de animales metazoarios con mayor importancia en cuanto a biomasa, variedad genética e interacciones bióticas en ecosistemas terrestres (Bautista et al. 2004; Grimaldi y Engel 2005).

En particular, los coleópteros son de gran interés debido a que poseen un potencial alto como bioindicadores del cambio y degradación del ambiente (Barbosa y Marquet 2002); han desarrollado la mayor diversidad estructural, dado su adaptación a una amplia variedad de ambientes; cumplen un rol de gran valor en la naturaleza, ya que actúan como recicladores de materia orgánica; mientras otros favorecen en gran medida a la polinización (Ciesla 2011), y pueden formar parte de la alimentación base de una vasta cantidad de seres vivos en las cadenas tróficas (Haskell 2000; Hunter 2002). Pocos estudios han abordado características del hábitat y su relación con la estructura del gremio de insectos para evaluar los efectos de la antropización en los humedales (Pauchard et al. 2006; Villagrán et al. 2006). En ecosistemas de humedales se ha sugerido una relación positiva entre la riqueza de especies de insectos y la naturalidad de la matriz, la heterogeneidad de la vegetación, la profundidad y la calidad del agua (Figueroa $e t$ al. 2003; Villagrán et al. 2006).

Por lo anterior, este estudio tuvo por objetivos: a) Analizar la variación estacional de la diversidad (abundancia y riqueza) de coleópteros epigeos (que viven en el suelo) en la ribera de la Laguna Carén, Santiago, Chile, y b) Evaluar parámetros físico- químicos, indicadores de la calidad del agua del cuerpo lacustre. Esto con el fin de aportar al conocimiento sobre la diversidad de estos coleópteros epigeos y su uso potencial como bioindicadores de la calidad del agua en cuerpos lacustres, actuando como herramienta complementaria al método tradicional de análisis físico-químico. Por último, cabe destacar que este trabajo constituye el primer registro de la entomofauna en esta laguna, conformada mayoritariamente por formaciones de bosque esclerófilo de la zona central de Chile, fragmentadas fuertemente en estas últimas décadas.

\section{Materiales y métodos}

Área de estudio. El cuerpo de agua y humedal Laguna Carén se sitúa en comuna de Pudahuel $\left(33^{\circ} 25^{\prime} 48,55^{\prime \prime} \mathrm{N}\right.$, $70^{\circ} 50^{\prime} 26,60$ 'E), Santiago, Chile. Se conforma como un cuerpo de agua superficial suburbano con una geomorfología relativamente plana, con lomajes suaves (cerca de 10 ha). En un tramo de su recorrido se desprenden dos brazos y además tiene una serie de esteros pequeños que fluyen hacia la laguna en forma de abanico. Este sector tiene un clima templado mediterráneo semiárido, caracterizado por una estación seca y de radiación alta, y otra, con precipitaciones y nubosidad durante el invierno. El régimen térmico varía, en promedio, entre una máxima en enero de $30,6^{\circ} \mathrm{C}$ y una mínima en julio de $4,3{ }^{\circ} \mathrm{C}$ (Santibáñez y Uribe 1990). En la ribera se observan plantaciones de eucalipto (Eucalyptus globulus Labill), formaciones boscosas esclerófilas nativas: espino (Acacia caven (Mol.) Mol.), algarrobo (Prosopis chilensis (Molina) Stuntz), maitén (Maytenus boaria Molina) y de especies semileñosas y herbáceas: romerillo (Baccharis linearis (Ruiz \& Pav.) Pers.), totora (Typha angustifolia L.), junco (Scirpus sp.), quilo (Muehlenbeckia hastulata (J. E. Sm.) I. M. Johnst.), cardo penquero (Cynara cardunculus L.), cardo ne- gro (Carduus pycnocephalus L.), galega (Galega officinalis L.), entre otras. La fauna de la zona se compone de especies de anfibios, reptiles, aves, y mamíferos, en su mayoría nativos y es un gran atrayente turístico. Cabe destacar que este ecosistema representativo del bosque esclerófilo de la zona de clima mediterráneo de Chile central ha sufrido eventos de contaminación agroindustriales esporádicos que incrementan su vulnerabilidad.

Evaluación de parámetros físico-químicos de la calidad del agua. Se evaluaron parámetros indicadores de la calidad del agua en cuatro estaciones de muestreo (EM) escogidas al azar, en dos temporadas (primavera de 2015 y verano de 2016). Las EM fueron representativas de las coberturas vegetales (arbóreas, semileñosas y herbáceas): EM1 (alta: $>75$ $\%$ ), EM2 (media-alta: 51-75 \%), EM3 (media- baja: 20-50 $\%$ ) y EM4 (baja: < $20 \%$ ). Las distancias entre ellas variaron entre 300 a $1.000 \mathrm{~m}$, dependiendo de las coberturas. En cada una de éstas, a partir de una muestra de volumen $1 \mathrm{~L}$, se midieron in situ y a través de un medidor multiparámetro (WTW Multi 340i, Weilheim) los valores de los parámetros físico-químicos, tales como: $\mathrm{pH}$, conductividad eléctrica (CE) $(\mathrm{mS} / \mathrm{cm})$, oxígeno disuelto $(\mathrm{OD})(\mathrm{mg} / \mathrm{L})$, temperatura $\left(\mathrm{T}^{\circ}\right)\left({ }^{\circ} \mathrm{C}\right)$, salinidad $(\%)$ y sólidos totales disueltos (STD) $(\mathrm{mg} / \mathrm{L})$; mientras que la transparencia $(\mathrm{cm})$ se determinó con un Disco Secchi. En cada EM se obtuvo tres sub-muestras en tubos Falcon de $50 \mathrm{~mL}$ que se transportaron en frío (4 ${ }^{\circ} \mathrm{C}$ ) al Laboratorio de Criminalística Central de la Policía de Investigaciones de Chile (PDI) para un análisis del color (Pt$\mathrm{Co})$, turbiedad (FAU), nitrógeno total $(\mathrm{mg} / \mathrm{L})$ y fósforo total $(\mathrm{mg} / \mathrm{L})$. Se siguieron los métodos indicados por la normativa vigente en Chile (CONAMA 2004; Instituto Nacional de Normalización Chile (INN Chile) 1987, 1996). Cada EM se clasificó según su calidad en Clase Excepcional, 1, 2, 3 y 4, correspondiendo a excelente, muy buena, buena, regular y mala, respectivamente (CONAMA 2004). En la tipificación de la calidad del agua se tomaron en cuenta principalmente los parámetros de $\mathrm{T}^{\circ}$ del agua, $\mathrm{OD}, \mathrm{pH}$ y $\mathrm{CE}$, considerados fundamentales para el desarrollo de la vida acuática y por estar interrelacionados entre sí.

Evaluación de abundancia y riqueza de coleópteros epigeos de la ribera. Los muestreos de coleópteros epigeos se hicieron en la ribera en las mismas EM y temporadas indicadas. Se usaron trampas de intercepción que consistieron en recipientes plásticos $(1 \mathrm{~L})$, cada uno con $0,4 \mathrm{~L}$ de etanol $\left(96^{\circ}\right)$ al $50 \%$ y $0,4 \mathrm{~L}$ de una solución de agua con detergente. En cada EM y temporada se instalaron al azar, enterradas a ras de suelo, cinco trampas separadas al menos $5 \mathrm{~m}$ de la orilla de la laguna, y con una distancia mínima de $10 \mathrm{~m}$ entre sí (radio de captura de $5 \mathrm{~m})$. Las trampas (unidad experimental) $(n=40)$ se retiraron a los 15 días de ser instaladas. El contenido de cada trampa se guardó en frascos plásticos con etanol al $75 \%$ para la conservación de las muestras, debidamente rotuladas. Los coleópteros se identificaron y contaron bajo lupa estereoscópica, con el apoyo de claves de reconocimiento y comparaciones con colecciones entomológicas de referencia del Laboratorio de Entomología Forestal, Facultad de Ciencias Forestales y de la Conservación de la Naturaleza, Universidad de Chile y del Instituto de Entomología de la Universidad Metropolitana de Ciencias de la Educación, en Santiago. Con la información de las identificaciones taxonómicas de los coleópteros epigeos encontrados en cada EM y tempora- 
da se estimó el índice de diversidad alfa mediante el cálculo de la abundancia (número de individuos por ha) y la riqueza (número de especies por ha). Además, de acuerdo con información bibliográfica se clasificó cada especie conforme a su gremio trófico: fitófago, saprófago o depredador; estimando su abundancia por EM y temporada. Para evaluar diferencias entre EM se usó la prueba no paramétrica de Kruskal-Wallis (H') $(\mathrm{P}<0,05)$. En los casos que se encontraron diferencias significativas se realizó una prueba post hoc de Dunn de comparaciones múltiples. Para evaluar diferencias entre temporadas se usó la prueba de Mann-Whitney (U'). Ambas pruebas se hicieron con el programa para análisis estadístico InfoStat, versión 2008 (InfoStat 2008).

\section{Resultados}

Evaluación de parámetros de la calidad del agua. La Laguna Carén presentó una apariencia estética deteriorada, con aguas turbias y en algunas EM malos olores, proliferación de microalgas y resuspensión de sedimentos, principalmente en la zona de actividades recreativas cercana a la EM4, donde también se detectó una vegetación más rala y poco diversa. En verano, en general, disminuyó el flujo de la corriente de agua; aumentó la ocurrencia de: microalgas, espuma, aceites o grasas; se redujo el avistamiento de peces y aves acuáticas y hubo resuspensión de sedimentos. Se constató la existencia de un sistema de tratamiento de aguas servidas, donde la laguna era el cuerpo receptor. La calidad del agua en la laguna en verano fue inferior que en primavera, principalmente por los niveles de OD (en particular en la EM3), CE y STD; además, los niveles de fósforo total en ambas temporadas estuvieron muy sobre lo normal, como asimismo el nitrógeno total, en particular en la EM4. Por lo que este cuerpo de agua tiene características eutróficas, es decir, no es adecuado para la conservación de comunidades acuáticas (CONAMA 2004) (Tabla 1).

Abundancia y riqueza de especies de coleópteros epigeos de la ribera. En total se detectaron 19 especies (14 nativas), representadas por ocho familias con un total de 329 individuos de coleópteros epigeos de la ribera de la laguna. Con respecto a la abundancia de coleópteros, en primavera la EM1 registró una abundancia significativamente mayor que las otras tres estaciones (153\% más que la EM3) $(\mathrm{P}<0,002)$ (Tabla 2), coincidiendo con la EM de mejor calidad del agua (Clase 2: buena). En verano la situación fue diferente, ya que la EM3 obtuvo una abundancia superior a las demás estaciones $(\mathrm{P}<0,04)(190 \%$ más que la EM4). Entre temporadas se observaron diferencias en la abundancia en la EM3 $(\mathrm{P}<0,01)$ y EM2 ( $\mathrm{P}<0,007)$. Al comparar en general la abundancia obtenida en las temporadas, la de verano fue mayor $(\mathrm{P}>0,0003)$ a la de primavera, superándola en $102 \%$ (Tabla 2).

En relación con la riqueza de especies, en primavera las EM2 y EM3 tuvieron una riqueza superior que las otras dos estaciones, en particular la EM2 (64 \% más que la EM4) (P $<0,01)$. En cambio, en verano la EM1 y EM2 presentaron la mayor riqueza, en especial la EM1 (88 \% más que la EM4) $(\mathrm{P}<0,004)$. Entre temporadas, se observaron diferencias en la riqueza de la EM2 $(\mathrm{P}<0,03)$ y EM3 $(\mathrm{P}<0,01)$. Al cotejar globalmente las riquezas obtenidas por temporada, en primavera hubo una riqueza significativamente mayor $(\mathrm{P}>0,01)$ con respecto al verano, superándola en $21 \%$ (Tabla 2).

Análisis de gremios tróficos por temporada. En la temporada de primavera se observaron variaciones en los gremios tróficos entre las estaciones de muestreo. A nivel de fitófagos, la EM1 registró una abundancia significativamente mayor que las demás estaciones (320\% más que la EM4) $\left(\mathrm{H}^{\prime}=\right.$ 13,58; $\mathrm{P}<0,002)$. En el nivel de los depredadores, las EM3 y EM4 registraron un número significativamente mayor de individuos, en particular la EM4 (175 \% más que la EM1) $\left(\mathrm{H}^{\prime}=8,10 ; \mathrm{P}<0,02\right)$. Y en el nivel de los saprófagos, la EM1 registró un número de individuos significativamente superior que las otras estaciones (340 \% más que la EM4) $\left(\mathrm{H}^{\prime}=15,66\right.$; $\mathrm{P}<0,001$ ) (Tabla 2).

En la temporada de verano se observaron variaciones significativas de los gremios tróficos sólo en depredadores y saprófagos entre las estaciones de muestreo. A nivel de fitó-

Tabla 1. Parámetros físico-químicos evaluados y clasificación de la calidad del agua en la Laguna Carén (Santiago, Chile) por temporada y estación de muestreo (EM).

\begin{tabular}{|c|c|c|c|c|c|c|c|c|}
\hline \multirow{2}{*}{$\begin{array}{l}\text { Parámetros } \\
\text { físico-químicos }\end{array}$} & \multicolumn{4}{|c|}{ Primavera 2015} & \multicolumn{4}{|c|}{ Verano 2016} \\
\hline & EM1 & EM2 & EM3 & EM4 & EM1 & EM2 & EM3 & EM4 \\
\hline Temperatura $\left({ }^{\circ} \mathrm{C}\right)$ & 24,3 & 28,4 & 25,2 & 24,9 & 20,00 & 22,10 & 19,50 & 20,90 \\
\hline$\Delta \mathrm{T}^{\circ}\left({ }^{\circ} \mathrm{C}\right)$ & 1,4 & 2,7 & 0,5 & 0,8 & 0,6 & 1,4 & 1,1 & 0,2 \\
\hline $\mathrm{OD}^{1}(\mathrm{mg} / \mathrm{L})$ & 8,1 & 8,4 & 8,4 & 8,5 & 5,8 & 5,3 & 2,3 & 8,1 \\
\hline $\mathrm{pH}$ & 9,1 & 9,2 & 9,3 & 9,2 & 9,2 & 8,4 & 7,4 & 8,6 \\
\hline $\mathrm{CE}^{2}(\mu \mathrm{S} / \mathrm{cm})$ & 813,0 & 828,0 & 824,0 & 835,0 & $1.271,0$ & $1.222,0$ & 921,0 & $1.340,0$ \\
\hline Salinidad (\%) & 0,3 & 0,3 & 0,3 & 0,3 & 0,6 & 0,6 & 0,6 & 0,6 \\
\hline $\mathrm{STD}^{3}(\mathrm{mg} / \mathrm{L})$ & 824,0 & 832,0 & 826,0 & 834,0 & $1.272,0$ & $1.240,0$ & 832,0 & $1.344,0$ \\
\hline Transparencia $(\mathrm{cm})$ & 20,0 & 20,0 & 20,0 & 27,5 & 30,0 & 80,0 & 25,0 & 50,0 \\
\hline Color (Pt-Co) & 157,0 & 179,0 & 182,0 & 97,0 & 240,0 & 200,0 & 88,0 & 142,0 \\
\hline Turbiedad (FAU) & 12,0 & 14,0 & 17,0 & 7,0 & 25,0 & 23,0 & 8,0 & 13,0 \\
\hline Fósforo total (mg/L) & 200,0 & $1.000,0$ & 600,0 & 300,0 & 400,0 & 700,0 & $1.200,0$ & 300,0 \\
\hline Nitrógeno total $(\mathrm{mg} / \mathrm{L})$ & 400,0 & 400,0 & 200,0 & $1.300,0$ & 500,0 & 200,0 & 500,0 & $1.500,0$ \\
\hline Clase calidad $^{4}$ & 2 & 3 & 2 & 3 & 3 & 2 & 3 & 3 \\
\hline
\end{tabular}

${ }^{1}$ Oxígeno disuelto; ${ }^{2}$ Conductividad eléctrica; ${ }^{3}$ Sólidos totales disueltos; ${ }^{4}$ Clase 2: buena, Clase 3: regular. 
Tabla 2. Abundancia (número de individuos por ha) de coleópteros epigeos en la ribera de la Laguna Carén (Santiago, Chile) por temporada y estación de muestreo (EM).

\begin{tabular}{|c|c|c|c|c|c|c|c|c|}
\hline \multirow{2}{*}{ Familia/Especie* } & \multicolumn{4}{|c|}{ Primavera de 2015} & \multicolumn{4}{|c|}{ Verano de 2016} \\
\hline & EM1 & EM2 & EM3 & EM4 & EM1 & EM2 & EM3 & EM4 \\
\hline \multicolumn{9}{|l|}{ Anthicidae } \\
\hline Anthicus floralis (Linnaeus, 1758) ${ }^{\mathrm{f}}$ & 0 & 0 & 0 & 76,4 & 0 & 0 & 90,0 & 0 \\
\hline \multicolumn{9}{|l|}{ Bostrichidae } \\
\hline Dexicrates robustus $\left(\right.$ Blanchard, 1851) ${ }^{\mathrm{f}}$ & 0 & 101,9 & 0 & 0 & 0 & 127,3 & 76,4 & 0 \\
\hline \multicolumn{9}{|l|}{ Buprestidae } \\
\hline Neocypetes guttulata (Fairmaire \& Germain, 1858) ${ }^{\mathrm{f}}$ & 50,9 & 50,9 & 0 & 0 & 0 & 0 & 0 & 0 \\
\hline Mastogenius sulcicollis (Philippi \& Philippi, 1864) ${ }^{\mathrm{f}}$ & 0 & 0 & 0 & 0 & 25,5 & 0 & 0 & 0 \\
\hline \multicolumn{9}{|l|}{ Carabidae } \\
\hline Tetragonoderus viridis (Dejean, 1831) ${ }^{\mathrm{d}}$ & 0 & 50,9 & 0 & 76,4 & 0 & 0 & 0 & 0 \\
\hline Parhypates sp. ${ }^{\mathrm{d}}$ & 0 & 0 & 101,9 & 0 & 0 & 0 & 0 & 0 \\
\hline Metius femoratus (Dejean, 1828) ${ }^{\mathrm{d}}$ & 0 & 0 & 0 & 127,3 & 0,0 & 0 & 50,9 & 356,5 \\
\hline Pachymorphus chalceus (Dejean, 1828) ${ }^{\mathrm{d}}$ & 0 & 0 & 0 & 25,5 & 76,4 & $1.095,0$ & $1.858,9$ & 0 \\
\hline \multicolumn{9}{|l|}{ Coccinellidae } \\
\hline Eriopis connexa chilensis $\left(\right.$ Hofmann, 1970) ${ }^{\mathrm{d}}$ & 0 & 0 & 101,9 & 50,9 & 76,4 & 50,9 & 0 & 0 \\
\hline Hippodamia variegata $(\text { Goeze, } 1777)^{\mathrm{d}}$ & 0 & 50,9 & 0 & 0 & 25,5 & 50,9 & 0 & 0 \\
\hline \multicolumn{9}{|l|}{ Histeridae } \\
\hline Euspilotus bisignatus (Erichson, 1834) ${ }^{\mathrm{s}}$ & 50,9 & 178,3 & 101,9 & 0 & 101,9 & 0 & 25,5 & 0 \\
\hline \multicolumn{9}{|l|}{ Protocucujidae } \\
\hline Ericmodes sylvaticus (Philippi \& Philippi, 1864) ${ }^{\mathrm{f}}$ & 483,8 & 0 & 0 & 50,9 & 0 & 0 & 0 & 0 \\
\hline \multicolumn{9}{|l|}{ Staphylinidae } \\
\hline Creophilus erythrocephalus (Fabricius, 1775) ${ }^{\mathrm{s}}$ & 0 & 101,9 & 0 & 0 & 0 & 0 & 0 & 0 \\
\hline Aleochara lata (Gravenhorst, 1802) ${ }^{\mathrm{s}}$ & 25,5 & 0 & 0 & 0 & 0 & 0 & 25,5 & 0 \\
\hline Glypholoma sp. ${ }^{\mathrm{s}}$ & 0 & 25,5 & 0 & 0 & 0 & 0 & 25,5 & 382,0 \\
\hline \multicolumn{9}{|l|}{ Tenebrionidae } \\
\hline Nycterinus substriatus (Solier, 1848) ${ }^{\mathrm{s}}$ & 458,4 & 101,9 & 127,3 & 127,3 & 942,2 & 101,9 & 76,4 & 0 \\
\hline Tribolium confusum (Jaquelin Du Val, 1868) ${ }^{\mathrm{f}}$ & 0 & 50,9 & 0 & 0 & 0 & 0 & 0 & 0 \\
\hline Praocis costatula (Solier, 1840) ${ }^{\mathrm{s}}$ & 25,5 & 0 & 0 & 0 & 0 & 0 & 0 & 0 \\
\hline Aspidolobus piliger (Redtenbacher, 1868) ${ }^{\mathrm{s}}$ & 0 & 0 & 0 & 0 & 50,9 & 0 & 0 & 0 \\
\hline \multirow[t]{2}{*}{$\mathrm{N}^{o}$ promedio de individuos por ha* } & $1.095,0$ & 713,0 & 432,9 & 534,8 & $1.298,7$ & $1.426,0$ & $2.139,0$ & 738,5 \\
\hline & $\mathrm{Ca}$ & $\mathrm{BCa}$ & Aa & $\mathrm{ABa}$ & $\mathrm{ABa}$ & $\mathrm{ABb}$ & $\mathrm{Bb}$ & $\mathrm{Aa}$ \\
\hline \multirow[t]{2}{*}{$\mathrm{N}^{o}$ promedio de especies por ha } & 331,0 & 458,4 & 407,4 & 280,1 & 382,0 & 356,5 & 280,1 & 203,7 \\
\hline & $\mathrm{ABa}$ & $\mathrm{Bb}$ & $\mathrm{Bb}$ & Aa & $\mathrm{Ba}$ & $\mathrm{Ba}$ & $\mathrm{ABa}$ & $\mathrm{Aa}$ \\
\hline \multirow[t]{2}{*}{$\mathrm{N}^{\circ}$ promedio de individuos fitófagos por ha } & 534,8 & 203,7 & 0 & 127,3 & 25,5 & 127,3 & 76,4 & 0,0 \\
\hline & $\mathrm{Cb}$ & $\mathrm{Bb}$ & Aa & $\mathrm{ABb}$ & $\mathrm{Aa}$ & Aa & Aa & $\mathrm{Aa}$ \\
\hline \multirow[t]{2}{*}{$\mathrm{N}^{\circ}$ promedio de individuos depredadores por ha } & 0 & 101,9 & 203,7 & 208,1 & 178,3 & $1.196,8$ & $1.909,9$ & 356,5 \\
\hline & Aa & $\mathrm{ABa}$ & $\mathrm{Ba}$ & $\mathrm{Ba}$ & $\mathrm{Ab}$ & $\mathrm{BCb}$ & $\mathrm{Cb}$ & $\mathrm{ABa}$ \\
\hline \multirow[t]{2}{*}{$\mathrm{N}^{\circ}$ promedio de individuos saprófagos por ha } & 560,2 & 407,4 & 229,2 & 127,3 & $1.095,0$ & 101,9 & 152,8 & 382,0 \\
\hline & $\mathrm{Ca}$ & $\mathrm{BCb}$ & $\mathrm{ABa}$ & Aa & $\mathrm{Bb}$ & $\mathrm{Aa}$ & Aa & $\mathrm{ABb}$ \\
\hline
\end{tabular}

* Gremios tróficos: ${ }^{\mathrm{f}}$ Fitófago, ${ }^{\mathrm{s}}$ saprófago, ${ }^{\mathrm{d}}$ depredador. $* *$ Letras mayúsculas y minúsculas distintas en cada fila indican diferencias significativas entre estaciones de muestreo (EM) (Prueba Dunn) y temporadas (Prueba Mann-Whiney), respectivamente $(\mathrm{P} \leq 0,05)$.

fagos, la EM2 tendió a presentar una mayor abundancia (las diferencias no fueron significativas; $\left.\mathrm{H}^{\prime}=5,14 ; \mathrm{P}>0,08\right)$ en comparación con las tres estaciones restantes (400\% más que la EM2). Destacó el bajo número de fitófagos en las cuatro EM. En el nivel de los depredadores, la EM3 registró un número significativamente mayor de individuos que las otras tres estaciones $\left(971 \%\right.$ más que la EM1) $\left(\mathrm{H}^{\prime}=9,54 ; \mathrm{P}<0,02\right)$. $\mathrm{Y}$ en los saprófagos, la EM1 registró un número de indivi- duos mayor que las otras estaciones (975 \% más que la EM2) (Tabla 2).

\section{Discusión}

De la calidad del agua en las EM analizadas en la Laguna Carén durante la primavera de 2015 y verano de 2016, se puede deducir que empeoró en esta última dado que los 
niveles de concentración promedio en la CE y de STD se elevaron, los de OD se redujeron y los de nitrógeno y principalmente fósforo total se incrementaron. Además, el flujo menor en la corriente del agua y el aumento en la proliferación de microalgas, decrecimiento de fauna (peces y aves acuáticas), aparición de espuma y aceites o grasas en la orilla de la laguna, podrían constituir un indicio de eutrofización (Quirós 2000).

El sector con mayor sedimento (EM4) era de acceso público y, en términos de masa total, la mayor fuente de contaminación del agua. La turbidez detectada que causó estos sedimentos pudo destruir sitios de alimentación o desove de peces, o bien, favorecer la proliferación de bacterias y agotar el OD, reduciendo la vida animal, tal como lo señalado por Moreno et al. (2010). El aporte excesivo de nutrientes, como el fósforo y nitrógeno, pudo dar lugar a una rápida proliferación de microalgas y vegetación acuática. Como consecuencia, el OD de la laguna se redujo sobre todo en la superficie, capa más oxigenada debido a su contacto con la atmósfera. Cuando estas microalgas y otros vegetales mueren, los microorganismos que los descomponen agotan el OD y se hace imposible la vida de otros seres vivos. El resultado es un agua maloliente e inutilizable (Mazzeo et al. 2002; Quirós 2000), como se observó particularmente en la EM4. Los niveles de nitrógeno y fósforo elevados registrados en las aguas de la laguna podrían explicarse por el vertido de aguas servidas, o bien en grandes extensiones, por ejemplo, por el escurrimiento de aguas ricas en fertilizantes agrícolas (Quirós 2000).

Probablemente la abundancia y riqueza superiores de las EM1, EM2 y EM3 pueden atribuirse a las mejores condiciones de hábitat con mayor disponibilidad de alimento (cobertura vegetal) junto con el aporte de los recursos tróficos necesarios para sobrevivir y establecerse (Correa y Roa 2005; Grez et al. 2003; Ramírez et al. 2017). Cabe hacer notar que la abundancia como la riqueza en la EM4 fueron inferiores en las dos temporadas, coincidiendo con una calidad del agua regular (Clase 3), posiblemente debido a su mayor grado de antropización, con la concentración de la mayoría de las actividades de recreación y menos disponibilidad de recursos vegetales.

Los tenebriónidos constituyen una de las familias más abundantes y diversas de los artrópodos y desempeñan un rol clave en los procesos de fragmentación biológica del recurso vegetal, en los ciclos de los nutrientes y en la dieta de otros organismos (vertebrados) consumidores (Adamopoulou y Legakis 2002; Cepeda et al. 2005; Orgeas et al. 2003). Su abundancia en las tres primeras EM se puede deber a la disponibilidad de hojarasca, un sustrato que proporciona condiciones ideales para sus ciclos de vida (ovipostura, desarrollo hipogeo de los estados larvarios, obtención de alimento, entre otros) (Pizarro et al. 2011). En cuanto a los carábidos, Canepuccia et al. (2009), Porrini et al. (2014), Purtauf et al. (2005) han comprobado que la calidad y estructura de la vegetación, el grado de antropización, entre otros, determinan los patrones de su distribución espacial; además, las precipitaciones afectan a estas especies, en particular a los depredadores y omnívoros, principalmente por la reducción del hábitat y de las presas disponibles; tal como la especie subxerófila Pachymorphus chalceus (Dejean, 1828) (Coleoptera: Carabidae), cuya abundancia aumentó en verano. La familia Protocucujidae representada por la única especie fitófaga Ericmodes sylvaticus (Philippi \& Philippi, 1864), pudo ver reflejada su abundancia alta por la disponibilidad de recursos tróficos. Por otro lado, el hecho que la abundancia varíe entre ambas temporadas se pudo deber a que algunas especies son univoltinas, mientras otras pudieron cumplir varias generaciones en un año. Un ejemplo es Nycterinus substriatus (Solier, 1848) (Tenebrionidae), una de las especies más abundantes cuyo ciclo de vida no es estacional (Saiz et al. 1989; Vidal y Guerrero 2007).

En primavera las condiciones invernales atípicas registradas en esta evaluación pudieron generar niveles mayores de pudrición y degradación del material vegetal, por lo que, en las tres primeras EM, donde se detectó una mayor cantidad de vegetación, coincidió también con una marcada presencia de coleópteros saprófagos (Ramírez et al. 2017). La abundancia de saprófagos puede explicarse por la carga de materia orgánica, que es retenida por la vegetación emergente en la laguna; ya que, no existe una mayor presencia de animales que puedan aportar este tipo de materia (Saiz et al. 1989; Solervicens 2014).

En verano se registró una fuerte participación de coleópteros depredadores en las EM2 y EM3, lo que puede deberse a que al aumentar la vegetación existe una mayor disponibilidad de refugios y alimento, que se traduce en que los insectos depredadores tengan más recursos para cubrir sus necesidades (Ramírez et al. 2017). Cepeda et al. (2005) y Baloriani et al. (2009) señalan que la abundancia de insectos depredadores es una consecuencia directa de la de fitófagos, por lo que la menor ocurrencia de estos últimos en verano se podría explicar por la aparición de los primeros (Fernández et al. 2014). En las EM1 y EM4 se registró una dominancia de insectos saprófagos, lo que podría deberse principalmente a la alta disponibilidad de alimento (material vegetal degradado). Cabrera (2012) señala que los insectos saprófagos son más abundantes y diversos en ambientes con una incorporación de hojarasca variada y continua, humedad en el suelo alta y temperaturas bajas, propias de las dos estaciones debido a las formaciones boscosas dominantes.

Por otro lado, la homogeneidad ambiental brindada en este tipo de ambientes caracterizada por el tipo de vegetación descrita, de gran cobertura, que se desarrolla en lugares que reciben un aporte constante de agua que aseguran condiciones estables y continua de temperatura y humedad adecuadas, es probable que actúe como factor de concentración de fauna que favorezca su diversidad. Por último, cabe considerar que los insectos son muy vulnerables a la fragmentación y pérdida del hábitat, donde los coleópteros son los más abundantes y cumplen un rol fundamental en procesos ecológicos tales como herbivoría, depredación o descomposición, por lo tanto, cambios en su composición o abundancia, producto de la fragmentación y pérdida de hábitat, pueden afectar el funcionamiento normal del ecosistema (Tscharntke et al. 2002).

\section{Conclusiones}

Este trabajo constituye el primer registro de especies de insectos de la Laguna Carén el que estuvo formado principalmente por especies nativas. La riqueza como la abundancia de coleópteros epigeos varió por temporada, siendo superior en sitios con mayor cobertura vegetal. La disponibilidad de alimento y refugio para los coleópteros epigeos estaría influenciada principalmente por factores antrópicos, que conducirían a un mayor grado de fragmentación y alteración del 
hábitat. La información generada podría servir como base para futuras investigaciones sobre bioindicadores en humedales vulnerables de la región mediterránea.

\section{Agradecimientos}

Fondo de Apoyo para Publicaciones Científicas, Departamento de Silvicultura y Conservación de la Naturaleza, Universidad de Chile.

\section{Literatura citada}

ADAMOPOULOU, C.; LEGAKIS, A. 2002. Diet of a lacertid lizard (Podarcis milensis) in an insular dune ecosystem. Israel Journal of Zoology 48: 207-219.

BALORIANI, G.; PALEOLOGOS, M. F.; MARASAS, M. E.; SARANDON, S. J. 2009. Abundancia y riqueza de la macrofauna edáfica (Coleoptera y Araneae), en invernáculos convencionales y en transición agroecológica. Revista Brasileira de Agroecologia 4 (2): 1733-1737.

BARBOSA, O.; MARQUET, P. A. 2002. Effects of forest fragmentation on the beetle assemblage at the relict forest of Fray Jorge, Chile. Oecologia 132: 296-306.

BAUTISTA, F.; PALACIO, J. E.; DELFÍN, H. 2004. Técnicas de muestreo para manejadores de recursos naturales. Consejo Nacional de Ciencia y Tecnología. Instituto Nacional de Ecología. Universidad Nacional Autónoma de México. D. F., México. 513 p.

CABRERA, G. 2012. La macrofauna edáfica como indicador biológico del estado de conservación/perturbación del suelo. Resultados obtenidos en Cuba. Pastos y Forrajes 35 (4): 349-364.

CANEPUCCIA, A. D.; CICCHINO, A. C.; ESCALANTE, A.; NOVARO, A.; ISAACH, J. P. 2009. Differential responses of marsh arthropods to rainfall-induced habitat loss. Zoological Studies 48 (2): 174-183.

CEPEDA, J.; PIZARRO, J.; VÁSQUEZ, H. 2005. Variación en la abundancia de Arthropoda en un transecto latitudinal del desierto costero transicional de Chile, con énfasis en los tenebriónidos epigeos. Revista Chilena de Historia Natural 78: 651-663.

CIESLA, W. 2011. Forest insect management in forest entomology: A global perspective. Editorial Wiley-Blackwell. Chichester, Reino Unido. $400 \mathrm{p}$.

CONAMA (Comisión Nacional del Medio Ambiente). 2004. Guía para el establecimiento de las normas secundarias de calidad ambiental para aguas continentales superficiales y marinas. Santiago, Chile. $18 \mathrm{p}$.

CORREA, P.; ROA, A. 2005. Relaciones tróficas entre Oncifelis guigna, Lycalopex culpaeus, Lycalopex griseus y Tyto alba en un ambiente fragmentado de la zona central de Chile. Mastozoología Neotropical 12: 57-60.

FERNÁNDEZ, I.; FAVILA, M. E.; LÓPEZ, G. 2014. Composición, riqueza y abundancia de coleópteros (Coleoptera) asociados a bosques semideciduos y vegetaciones rudelares en la Sierra del Rosario, Cuba. Boletín de la Sociedad Entomológica Aragonesa 54: 329-339.

FIGUEROA, R.; VALDOVINOS, E.; ARAYA, E.; PARRA, O. 2003. Macroinvertebrados bentónicos como indicadores de calidad del agua de ríos del sur de Chile. Revista Chilena de Historia Natural 76: 275-285.

FIGUEROA, R.; SORIA, M.; BELTRÁN, M.; CORREA, F. 2016. Estudio de comunidades biológicas como bioindicadores de calidad de agua. pp. 23-34. En: Chatata B.; Talavera C.; Villasante F. (Eds.). Estudio de comunidades biológicas como bioindicadores de calidad de agua. $1^{\text {a }}$ Edición. Universidad Nacional de San Agustín-CONCYTEC. Arequipa, Perú.

GREZ, A. A.; MORENO, P.; ELGUETA, M. 2003. Coleópteros (Insecta: Coleoptera) epigeos asociados al bosque maulino y plantaciones de pino aledañas. Revista Chilena de Entomología 29: 9-18.

GRIMALDI, D.; ENGEL, M. S. 2005. Evolution of the insects. Nueva York, EE. UU.: Cambridge University Press.

HASKELL, D. 2000. Effects of forest roads on macroinvertebrate soil fauna of the southern Appalachian Mountains. Conservation Biology 14 (1): 57-63.

HUNTER, M. D. 2002. Landscape structure, habitat fragmentation, and the ecology of insects. Agricultural and Forest Entomology 4 (3): 159-166.

INFOSTAT. 2008. Programa InfoStat versión 2008. Grupo InfoStat, FCA, Universidad Nacional de Córdoba. Córdoba, Argentina.

Instituto Nacional de Normalización (INN CHILE). 1987. Norma Chilena Oficial de Requisitos de calidad del agua para diferentes usos NCh 1333. Of.78. Santiago, Chile. 9 p.

Instituto Nacional de Normalización (INN CHILE). 1996. Norma Chilena Oficial de Calidad de Agua NCh 411/1-4. Santiago, Chile.

MAZZEO, N.; CLEMENTE, J.; GARCÍA-RODRÍGUEZ, F.; GORGA, J.; KRUK, C.; LARREA, D.; MEERHOFF, M.; QUINTANS, F.; RODRÍGUEZ-GALLEGO, L.; SCASSO, F. 2002. Eutrofización: Causas, consecuencias y manejo. pp. 39-55. En: Domínguez, A.; Prieto, R. G. (Eds.). Perfil Ambiental del Uruguay. Nordan-Comunidad. Montevideo, Uruguay.

MORENO, D. P.; QUINTERO, J.; LÓPEZ, A. 2010. Métodos para identificar, diagnosticar y evaluar el grado de eutrofia. Revista ContactoS 78: 25-33.

ORGEAS, J.; VIDAL, E.; PONEL, P. 2003. Colonial seabirds change beetle assemblages on a Mediterranean island. Ecoscience 10: 38-44.

PAUCHARD, A.; AGUAYO, M.; PEÑA, E.; URRUTIA, R. 2006. Multiple effects of urbanization on the biodiversity of developing countries: the case of a fast-growing metropolitan area (Concepción, Chile). Biological Conservation 127: $272-281$

PIZARRO, J.; JEREZ, V.; CEPEDA, J.; ALFARO, F. M. 2011. Caracteres preimaginales y aspectos bionómicos de Gyriosomus luczotii Laporte, 1840 (Coleoptera: Tenebrionidae), elemento endémico y erémico del desierto costero chileno. Animal Biodiversity and Conservation 34 (2): 265-272.

PORRINI, D. P.; CASTRO, A. V.; CICCHINO, A. C. 2014. Los carábidos (Coleoptera: Carabidae) asociados a los remanentes de bosque nativo en la Reserva Natural Municipal Laguna de los Padres, Buenos Aires. Revista de la Sociedad Entomológica Argentina 73 (1-2): 35-48.

PURTAUF, T.; DAUBER, J.; WOLTERS, V. 2005. The response of carabid beetles to landscape differs between trophic groups. Oecologia 142: 458-464.

QUIRÓS, R. 2000. La eutrofización de las aguas continentales de Argentina. pp. 43-47. En: Fernández, A. (Ed.). El agua en Iberoamérica: Acuíferos, lagos y embalses. CYTED. Subprograma XVII. Aprovechamiento y Gestión de Recursos Hídricos. Universidad de Buenos Aires, Facultad de Agronomía. Mar del Plata, Argentina. $147 \mathrm{p}$.

RAMÍREZ, P.; HUERTA, A.; ARAYA J. E. 2017. Effect of postharvest forestry residue management practices on the diversity of epigeal coleopterans. Revista Facultad Nacional de Agronomía 70 (1): 8069-8075.

SAIZ, F.; SOLERVICENS, J.; OJEDA, P. 1989. Coleópteros del Parque Nacional La Campana y de Chile Central. Universidad Católica de Valparaíso. Ediciones Universitarias de Valparaíso. Valparaíso, Chile. $124 \mathrm{p}$

SANTIBÁÑEZ, F.; URIBE, J. M. 1990. Atlas Agroclimático de Chile: Regiones V y Metropolitana. Universidad de Chile, Facultad de Ciencias Agrarias y Forestales, Laboratorio de Agroclimatología, Santiago, Chile. 66 p.

SOLERVICENS, J. 2014. Coleópteros de la Reserva Nacional Río Clarillo, en Chile central: taxonomía, biología y biogeografía. Universidad Metropolitana de la Educación, Corporación Na- 
cional Forestal, Gerencia de Áreas Silvestres Protegidas. Santiago, Chile. 478 p.

TENJO, A. I.; CÁRDENAS, E. 2015. Importancia y utilidad de los bioindicadores acuáticos. Diversidad Colombia. Revista de Investigación Científica 5: 39-48.

TSCHARNTKE, T.; STEFFAN-DEWENTER, I.; KRUESS, A.; THIES, C. 2002. Contribution of small habitat fragmentation to conservation of insect communities on grassland-cropland landscapes. Ecological Applications 12: 354-363.

VIDAL, P.; GUERRERO, M. 2007. Los tenebriónidos de Chile. Ediciones Universidad Católica de Chile. Santiago, Chile. $478 \mathrm{p}$.

VILLAGRÁN, R.; AGUAYO, M.; PARRA, L. E.; GONZÁLEZ, A. 2006. Relación entre características del hábitat y estructura del ensamble de insectos en humedales palustres urbanos del centro-sur de Chile. Revista Chilena de Historia Natural 79: 195-211.
WWDR (The World Water Development Report). 2015. Agua para un mundo sostenible. UNESCO (Organización de las Naciones Unidas para la Educación, la Ciencia y la Cultura), Perugia. Disponible en: http://www.unesco.org/new/fileadmin/ MULTIMEDIA/HQ/SC/images/WWDR2015ExecutiveSummary_SPA_web. pdf. [Fecha revisión: 26 junio 2017].

Recibido: 5-nov-2017 • Aceptado: 25-oct-2018

Citación sugerida:

ALVARADO-ORELLANA, A.; HUERTA-FUENTES, A.; PALMA-MUÑOZ, A.; RODRÍGUEZ-TOBAR, S. 2018. Variación estacional de la diversidad de coleópteros epigeos en la Laguna Carén (Santiago, Chile). Revista Colombiana de Entomología 44 (2): 266-272. Julio - Diciembre 2018. 